\title{
Elevated systemic expression of ER stress related genes is associated with stress-related mental disorders in the Detroit Neighborhood Health Study
}

\author{
Lisa Nevell ${ }^{a}$, Kezhong Zhang ${ }^{a, b}$, Allison Aiello ${ }^{c}$, Karestan Koenen ${ }^{d}$, Sandro Galea ${ }^{e}$, Richelo \\ Soliven $^{\mathrm{a}}$, Chao Zhang ${ }^{\mathrm{a}}$, Derek E. Wildman ${ }^{\mathrm{a}, \mathrm{f}}$, and Monica Uddin ${ }^{\mathrm{a}, \mathrm{g},{ }^{*}}$ \\ aWayne State University School of Medicine, Center for Molecular Medicine and Genetics, Detroit \\ MI 48201 USA \\ bWayne State University School of Medicine, Department of Immunology and Microbiology, \\ Detroit MI 48201 USA \\ 'University of Michigan School of Public Health, Ann Arbor, MI 48109 USA \\ ${ }^{d}$ Columbia University Mailman School of Public Health, Department of Epidemiology, New York \\ NY 100 USA \\ eColumbia University, Department of Epidemiology, New York NY 10032 USA \\ fWayne State University School of Medicine, Department of Obstetrics and Gynecology, Detroit \\ MI 48201 USA
}

gWayne State University School of Medicine, Department of Psychiatry and Behavioral Neurosciences, Detroit MI 48201 USA

\section{Abstract}

Background-The role of Endoplasmic Reticulum (ER) stress response in mental illness is not well understood. Human studies and animal models of depression show elevated brain ER stress

\footnotetext{
(C) 2014 Elsevier Ltd. All rights reserved.

"Corresponding Author: Monica Uddin, PhD., Assistant Professor, Center for Molecular Medicine and Genetics, Department of Psychiatry \& Behavioral Neurosciences, Wayne State University School of Medicine, 540 E. Canfield Ave, Detroit, MI 48201 USA [Ph 313-577-3265] monica.uddin@wayne.edu.

Disclosures

For work unrelated to this project, Sandro Galea is funded in part by a grant from Merck Pharmaceuticals; Derek Wildman is funded in part from Lung, LLC, receives Honoraria from Elsevier, INC; and Allison Aiello consults for SCA Tork. Lisa Nevell, Kezhong Zhang, Karestan Koenen, Richelo Soliven, Chao Zhang, and Monica Uddin declare no conflict of interest. This study was supported by the National Institutes of Health (DA022720, DA022720-S1, and RC1MH088283); the Wayne State University School of Medicine Post-doctoral Research Fellowship; the American Association of Anatomists Post-doctoral Fellowship; and the National Science Foundation (BCS 0827546).

Conflict of Interest Statement

For work unrelated to this project, Sandro Galea is funded in part by a grant from Merck Pharmaceuticals; Derek Wildman is funded in part from Lung, LLC, receives Honoraria from Elsevier, INC; and Allison Aiello consults for SCA Tork. Lisa Nevell, Kezhong Zhang, Karestan Koenen, Richelo Soliven, Chao Zhang, and Monica Uddin declare no conflict of interest.

Publisher's Disclaimer: This is a PDF file of an unedited manuscript that has been accepted for publication. As a service to our customers we are providing this early version of the manuscript. The manuscript will undergo copyediting, typesetting, and review of the resulting proof before it is published in its final citable form. Please note that during the production process errors may be discovered which could affect the content, and all legal disclaimers that apply to the journal pertain.
} 
response. In addition, some ER stress associated disorders (e.g. cardiovascular disease) show higher rates of depression compared to the general population, raising the possibility that ER stress response contributes to depression risk. It remains unknown, however, if ER stress response is present among individuals suffering from other stress-related mental illness, and whether such a response would be evident in a non-clinical sample. This study tests for systemic changes in ER stress response associated with major depressive disorder (MDD) or post-traumatic stress disorder (PTSD) among community-dwelling individuals.

Methods-We analyzed expression of BiP, EDEM1, CHOP, and XBP1, the major indicators of ER stress response, with Real-Time PCR in leukocyte-derived RNA samples from 86 participants of the Detroit Neighborhood Health Study. Participants were selected based on the presence of either past year MDD or past year PTSD; controls were age and sex matched.

Results-Relative to controls, MDD is associated with a 1.34-fold increase in $\operatorname{BiP}(\mathrm{P}=0.004)$, 1.35-fold increase in EDEMI ( $\mathrm{P}=0.001), 1.68$-fold increase in $C H O P(\mathrm{P}=0.002)$, and 1.60-fold increase in $X B P 1(\mathrm{P}=0.004)$. These results remained significant after correction for multiple testing. In contrast, PTSD is associated with a 1.27 fold increase in EDEMI expression only $(\mathrm{P}=0.027)$, a result that is attenuated to non-significance following adjustment for multiple testing; however, a subsample of participants with past month PTSD showed elevated expression of BiP and EDEMI (uncorrected p value 0.049 and 0.017, respectively).

Conclusions-These data indicate systemic and persistent activation of the ER stress response pathway in MDD among community-dwelling individuals. Systemic activation of the ER stress response may also occur in PTSD among persons with more recent symptoms.

\section{Keywords}

Endoplasmic Reticulum Stress; Unfolded Protein Response; Epidemiology; Matched-Pair Analysis; Case Control Studies; Gene Expression Pattern Analysis; Cardiovascular Diseases; Metabolic Diseases

\section{Introduction}

Several human studies and animal models suggested that endoplasmic reticulum (ER) stress response may play a role in psychiatric disease (Bown et al., 2000; Gold et al., 2013). The ER is an intracellular organelle that is responsible for protein folding and assembly, calcium storage, and lipid and sterol biosynthesis (Back et al., 2005). A variety of pharmacological, pathophysiological, and environmental stimuli can impose stress on the ER and subsequently interrupt the protein folding process in the ER, leading to accumulation of unfolded or misfolded proteins in the ER lumen (Zhang and Kaufman, 2004). This condition is referred to as "ER stress" (Zhang and Kaufman, 2004). To cope with ER stress, highly specific signaling pathways localized to the ER have evolved, which are collectively called the ER stress response or the "Unfolded Protein Response (UPR)". The primary function of the UPR is to restore ER homeostasis and help the cells adapt to ER stress conditions. However, when ER stress is prolonged or the degree of ER stress is too severe, UPR signaling can initiate programmed cell death by activating stress-induced pro-apoptotic factors (Zhang and Kaufman, 2004). Dysregulation or hyper-activation of the UPR pathway is critically involved in the initiation and progression of a variety of life-threatening 
diseases, such as cardiovascular disease, metabolic disease, neurodegenerative disease, and cancer (Zhang and Kaufman, 2008).

ER stress related protein expression has been shown to be elevated within the temporal cortex among individuals who had Major Depressive Disorder (MDD) and died of suicide compared with individuals who had MDD and died of other causes (Bown et al., 2000). A recent paper suggests that ER stress and parainflammation-i.e. a tissues' stress response with features intermediate between a normal state and an acute inflammatory state-are interrelated processes each influencing many phases of the stress response pathway and as such these pathways may be valid targets for intervention in MDD and bipolar disorder (Gold et al., 2013). Some pharmacological interventions used to treat affective disorders target genes which interact with the UPR pathways (e.g. Wang et al., 1999). For example, valproate and carbamazepine are mood stabilizing drugs which increase expression of 78kilodalton glucose-regulated protein (GRP78), also known as Binding immunoglobulin protein (BiP) (Wang et al., 1999). BiP/GRP78 is a member of the ER stress gene family believed to inhibit ER stress response activation and to inhibit UPR induced apoptosis (Reddy et al., 2003). Furthermore, lithium is a treatment employed for some mood disorders and is known to directly target and inhibit the protein encoded by Glycogen synthase kinase-3 (GSK3); GSK3 has been shown to regulate ER stress induced apoptosis in neuronal cells (Meares et al., 2011). Several mouse models of depression-like behavior implicate genes which interact with the UPR pathways, e.g. Calreticulin (CALR)(Liu et al., 2011), Bax inhibitor 1(BAX)(Hunsberger et al., 2011), Glycogen synthase kinase-3 (GSK3B)(Mines et al., 2010; Meares et al., 2011), Interferon-gamma (IFNG)(O'Connor et al., 2009), and Tumor necrosis factor-a(TNF) (Kaster et al., 2012). These human studies and animal models suggest that the UPR pathways may be activated or up regulated within brain tissue in the presence of some affective disorders.

Despite the suggestive evidence of ER stress in mental disorders in both animal (Hunsberger et al., 2011; Liu et al., 2011)) and human (Bown et al., 2000; So et al., 2007) studies, to date there has to our knowledge been no report of ER stress and the UPR among living individuals suffering from such disorders. More specifically, it is not known whether the ER stress response pathway is activated at a systemic level among community-dwelling individuals suffering from MDD. Here we test whether ER stress-related gene expression is elevated in leukocytes derived from individuals diagnosed with MDD in the Detroit Neighborhood Health Study (DNHS). The DNHS is a longitudinal community-based study of mental and physical health and their interaction with social-environmental factors (Uddin et al., 2010, Goldmann et al., 2011). Elevated ER stress response may be specific to MDD or it may be associated with additional mental disorders in which stress plays a contributing role. To test this, we also investigated whether ER stress response-related gene expression is elevated in leukocytes derived from individuals diagnosed with post-traumatic stress disorder (PTSD) in the Detroit Neighborhood Health Study (DNHS). To our knowledge this is the first consideration of whether PTSD is associated with elevated ER stress response and is facilitated by the relatively high burden PTSD in the DNHS population (Uddin et al., 2010). Results from this work may provide insight into biological processes relevant to psychological stress that occur in non-clinical settings. 


\section{Methods}

\section{Sample}

Details of the Detroit Neighborhood Health Study (DNHS) have been previously reported (Uddin et al., 2010, Goldmann et al., 2011, Uddin et al., 2011). The University of Michigan IRB reviewed and approved this study. Our study sample consisted of a subset of the DNHS study participants with previously extracted RNA from leukocytes (described in more detail below). We employed a matched pair study design based on past year MDD cases $(n=18)$ and their age- and gender-matched controls $(\mathrm{n}=18)$ and past year PTSD cases $(\mathrm{n}=26)$ and their age and gender-matched, trauma-exposed controls $(n=26)$. Note that two participants serve the role of matched control in both the MDD and PTSD control samples (total sample $\mathrm{n}=86$ ).

\section{Assessment of PTSD and MDD}

Past year MDD was assessed by a structured telephone interview using validated instruments based on DSM-IV criteria (APA, 1994). The Patient Health Questionnaire (PHQ-9)(Kroenke et al., 2001) was used to assess depression symptoms. The nine items on the PHQ-9 were scored from 0 (not at all) to 3 (nearly every day), with scores ranging from 0 to 27. In our MDD positive cases, the respondent experienced at least 5 symptoms more than half the time; this corresponds with a score of 10 out of 27 possible. Additional questions were added to these measures to determine the timing, duration and severity of illness and also symptom-related disability. We selected participants diagnosed with MDD during their lifetime who have also experienced symptoms during the past year. We selected age and sex matched control cases who have no lifetime history of MDD. We further excluded participants who have a lifetime history of PTSD as described below.

Past year PTSD was assessed in the following manner: Participants were asked to identify potentially traumatic events (PTEs) from a list of 19 categories of events. PTSD symptoms were assessed in reference to both the traumatic event the participant regards as their worst and one randomly selected traumatic event from the remaining traumas the participant has experienced. Lifetime PTSD cases met all six DSM-IV criteria in reference to either the worst or random traumatic event. After determining that a participant had a lifetime history of PTSD, we asked a follow up question regarding the length of time since an individual experienced any symptoms. We included only those individuals who have also experienced PTSD symptoms within the past year. We excluded participants with a history of lifetime MDD as described above. Finally, we identified a subset of these past year PTSD cases (and matched controls) who have experienced PTSD symptoms within the past month $(n=32)$. PTSD and depression diagnoses have been validated in a random subsample of participants via in-person clinical interview using the clinician-administered PTSD scale for DSM-IV and the structured clinical interview for DSM-IV disorders, respectively, as previously described (Uddin et al, 2010).

\section{Expression levels of ER stress related genes}

Eighteen $\mathrm{ml}$ of whole blood was collected from consenting DNHS participants. Total leukocyte RNA was isolated using the LeukoLOCK Fractionation and Stabilization kit 
(Ambion) using the manufacturer's alternative protocol. Quantitative reverse-transcriptase real time PCR (qRT-PCR) was used to quantify mRNA expression of ER stress-related genes (Zhang and Kaufman, 2004). These genes included: 1) BiP/GRP78, an abundant ER chaperone protein which has been recognized as a reliable ER stress marker and a master regulator of the UPR pathways (Zhang and Kaufman, 2004); 2) the growth arrest and DNA damage-inducible protein $G A D D 34 / C H O P$, an ER stress-induced pro-apoptotic factor under the UPR pathway mediated through PERK/eIF2 $a$ (Zhang and Kaufman, 2004). Induction of CHOP can represent induction of the PERK/eIF2 $a$ UPR pathways and ER stress-induced apoptosis (Zhang and Kaufman, 2004); 3) ER degradation enhancer, Mannosidase alpha-like 1 (EDEM1), an ER stress-inducible factor that is involved in ER-associated degradation (ERAD) of misfolded proteins (Molinari et al., 2003); and 4) The spliced form of X-box binding protein $1(X B P 1)$, an ER stress-induced bZIP transcription factor that is under the IRE $1 a$-mediated UPR pathways. Induction of the spliced XBP1 mRNA $(X B P 1 s)$ is an indication of activation of the IREI $/ X B P 1$ UPR pathway.

First strand cDNA was synthesized from 500ng of total RNA using the SuperScript III FirstStrand Synthesis SuperMix for qRT-PCR (Invitrogen). Primers targeting the following loci were used:

\section{BiP-F: 5'-CCTGGGTGGCGGAACCTTCGATGTG-3'}

BiP-R: 5'-CTGGACGGGCTTCATAGTAGACCGG-3'

CHOP-F: 5'-GCCTTTCTCCTTTGGGACACTGTCCAGC-3'

CHOP-R: 5'-CTCGGCGAGTCGCCTCTACTTCCC-3'

EDEM1-F: 5'-GCTACGACAACTACATGGCTC-3'

EDEMI-R: 5'-GACTTGGACGGTGGAATCTTT-3'

XBP1s-F: 5'-CCGC AGCAGGTGCAGG-3'

XBP1s-R: 5'-GAGTCAATACCGCCAGAATCCA-3'

beta-actin-F: 5'-AGCCTCGCCTTTGCCGATCCG-3'

beta-actin-R: 5'-ACATGCCGGAGCCGTTGTCGA-3'

Note that the sense primer for $X B P 1$ includes 4 bp before and 12 bp after a 26 bp region which is removed from exon 4 through non-conventional splicing during XBPI activation. Therefore this primer pair is expected to anneal only the spliced form of the gene; a similar strategy has been employed to investigate the function of the spliced form of XBPIin the

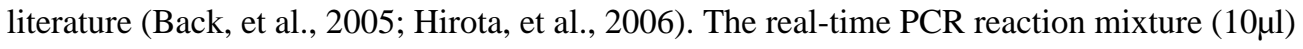
containing $125 \mathrm{nM}$ primers, cDNA template and SYBR Green PCR Master Mix (Invitrogen \# 4385612) was amplified in a 7500 Fast Real-time PCR System (Applied Biosystems). Cycling conditions included: holding stage at $95^{\circ} \mathrm{C}$ for 20 seconds; 40 cycling stages at $95^{\circ} \mathrm{C}$ for 3 seconds, $60^{\circ} \mathrm{C}$ for 30 seconds. Negative reverse transcriptase samples were used to ensure the absence of contaminating genomic DNA. All reactions were performed in triplicate. Each set of triplicates was checked to ensure that the threshold cycle $(\mathrm{Ct})$ values are all within $1 \mathrm{Ct}$ of each other. Fold changes were determined after normalizing to beta- 
actin as the internal control. The $2^{-\Delta \mathrm{Ct}}$ method (Livak and Schmittgen, 2001, Schmittgen and Livak, 2008) was employed to determine the relative expression values of the cases compared with age and sex matched controls. Normality was tested using a Shapiro-Wilk test. We tested for significant differences between case and control in the $2^{-\Delta \mathrm{Ct}}$ values using the non-parametric paired sample Wilcoxon signed rank test (P $₫ 0.05$ ) or the parametric paired samples $\mathrm{T}$ test, as appropriate. We tested which genes show a significant difference between cases and controls when a Bonferroni correction for multiple comparisons is employed (P $₫ 0.0125$ ).

\section{Prevalence of cardiovascular disease associated with ER stress in cases vs. controls}

Recent work has demonstrated that ER stress, and the consequent induction of the UPR plays a major role in the pathophysiology of cardiovascular (CVD)-related disorders such as cardiac hypertrophy, heart failure, atherosclerosis, and ischemic heart disease (Minamino and Kitakaze, 2010; Jiao et al., 2012). Therefore, as a secondary analysis, we tested whether there was a significant difference between cases and controls with respect to the prevalence of CVD. The prevalence of CVD was determined by assessing the presence/absence of conditions previously identified as indicative of CVD in the DNHS cohort (Keyes et al., 2013) and which have been previously associated with the ER stress response, including: hypertension (i.e. high blood pressure) (Sun et al., 2008), myocardial infarction (i.e. heart attack) (Thuerauf et al., 2006), stroke (Chae et al., 2004), chest pain due to heart disease (angina pectoris)(Myoishi et al., 2007), and congestive heart failure or heart disease (Hamada et al., 2004). Presence/absence of these diseases was assessed with a structured phone interview survey in which respondents were asked to respond "Has a doctor ever told you that you have [ITEM]?", with [item] referring to the four CVD-related conditions listed above. Cases meeting at least one of these criteria were identified as positive for CVD. Differences in the prevalence of CVD were tested using a McNemar's test (P $\unlhd$ (0.05), a paired version of the Chi-square test.

\section{Results}

Table 1 describes the demographic profile of the study participants. A Chi squared test shows no difference between case and control groups with respect to age, sex, or race/ ethnicity.

\section{Past year MDD is associated with induction of the ER stress response and activation of multiple UPR pathways in leukocyte samples (Figure 1)}

Among past year MDD cases, there is a 1.34 fold increase in expression of the ER chaperone $B i P\left(\mathrm{P}=0.004\right.$; mean \pm s.d. $2^{-\Delta \mathrm{CT}}$ for cases $0.0025 \pm 0.0014$ and controls 0.0019 $\pm 0.0009)$. The $E R A D$ mediator $E D E M 1$ also shows a 1.35 fold increase $(\mathrm{P}=0.001$; mean \pm s.d. $2^{-\Delta \mathrm{CT}}$ for cases $0.0125 \pm 0.0036$ and controls $0.0092 \pm 0.0026$ ) among MDD cases. In addition, there is a 1.68 fold increase in the expression of the ER stress-induced proapoptotic factor $C H O P\left(\mathrm{P}=0.002\right.$; mean \pm s.d. $2^{-\Delta \mathrm{CT}}$ for cases $0.0011 \pm 0.0007$ and controls $0.0006 \pm 0.0005)$. Finally, MDD cases show a 1.60 fold increase in expression of the spliced (i.e. activated) form of the UPR trans-activator $X B P 1$ ( $\mathrm{P}=0.004$; mean \pm s.d. $2^{-\Delta \mathrm{CT}}$ for cases $0.0327 \pm 0.0366$ and controls $0.0205 \pm 0.0245)$. Each of these genes remains significantly 
differently expressed between cases and controls when adjusted for multiple comparisons using the stringent Bonferroni correction (P $₫$ D.0125). Because our study sample included only 10 individuals with past month MDD, we did not assess ER stress activation for this phenotype.

\title{
Past year PTSD is associated with elevated expression of genes involved in ER-associated degradation (ERAD) of misfolded proteins in leukocyte samples (Figure 1)
}

\begin{abstract}
Among past year PTSD cases, EDEM1 shows a 1.27 fold increase ( $\mathrm{P}=0.027$; mean \pm s.d. $2^{-\Delta C T}$ for cases $0.0054 \pm 0.0039$ and controls $0.0036 \pm 0.0020$ ) in expression compared to controls. Expression of $\mathrm{BiP}$ also shows a 1.16 fold increase in cases that approaches significance $\left(\mathrm{P}=0.069\right.$; mean \pm s.d. $2^{-\Delta \mathrm{CT}}$ for cases $0.0048 \pm 0.0034$ and controls $0.0042 \pm$ 0.0028). In contrast to MDD cases, past year PTSD cases do not show a statistically significant increase in the expression of $C H O P$ or $X B P 1$ in cases compared with controls $(\mathrm{P}>0.05)$. When a conservative adjustment is made to account for multiple comparisons, i.e. the Bonferroni correction, EDEMI expression is no longer significantly different between cases and controls.
\end{abstract}

\section{Time since participant experienced PTSD symptoms does not alter the expression pattern of the ER stress-related genes (Figure 1)}

The expression levels of BiP and EDEMI are elevated in past month PTSD cases; similarly, $B i P$ and $E D E M 1$ expression levels are elevated in past year PTSD. Among PTSD cases who experienced symptoms recently (within the past month), there is a 1.25 fold increase in BiP expression $\left(\mathrm{P}=0.049\right.$; mean \pm s.d. $2^{-\Delta \mathrm{CT}}$ for cases $0.0036 \pm 0.0020$ and controls $0.0029 \pm$ $0.0017)$, and $E D E M 1$ also shows a 1.55 fold increase ( $\mathrm{P}=0.017$; mean \pm s.d. $2^{-\triangle \mathrm{CT}}$ for cases $0.0054 \pm 0.0050$ and controls $0.0034 \pm 0.0028$ ). The expression levels of $C H O P$ and $X B P I$ are not significantly different in cases compared with controls among either past month or past year PTSD cases. When a conservative adjustment is made to account for multiple comparisons, i.e. the Bonferroni correction, $B i P$ and $E D E M 1$ expression is no longer significantly differently expressed between cases and controls.

\section{Prevalence of cardiovascular disease in case and control groups}

The ER stress response is associated with CVD (Glembotski, 2007). Moreover, PTSD plays a role in increased risk and severity of cardiovascular disease (Kubzansky et al., 2007; Boscarino and Adams, 2008;,). Similarly, depression is a well-recognized risk factor for coronary artery disease (Grippo and Johnson, 2002). Consequently, we hypothesized a role for ER stress response in the comorbidity between mental disease and CVD and tested whether there were differences in the prevalence of this disorder among particpants with vs. without MDD or PTSD. No significant differences are found between our case and control groups according to a McNemar's Chi-squared test $\mathrm{P}>0.05$ [Table 2]. These data suggest that the differences shown in expression levels between cases and controls are likely due to psychological disease independent of CVD disease. 


\section{Discussion}

Here we report that community-dwelling individuals with a recent history of stress-related mental disorders show activation of the ER stress response and an increase in UPR related gene expression in peripheral leukocytes. The pattern of activation of the UPR pathways differed to some degree between MDD and PTSD, with MDD cases showing evidence of more pronounced ER stress response. In addition, the pattern of ER stress response was generally consistent in cases experiencing PTSD symptoms in the past month and the past year, indicating a persistent activation of the ERAD-associated UPR pathway among those suffering from this disorder. Secondary analyses of the prevalence of ER-stress associated CVD showed no difference between MDD- and PTSD- cases and their respective controls; consequently, differences observed between cases and controls are likely to be due specifically to the mental illness. Taken together, these results suggest that stress-related mental disorders, in particular MDD, are associated with a systemic, persistent ER stress response and activation of the UPR pathways.

The expression profiles observed in these data suggest both commonalities and dissimilarities in the pattern of ER stress response associated with MDD or PTSD. The ER stress marker $B i P$ is up-regulated in both MDD and in past month PTSD. BiP is transcriptionally activated by several mediators of the UPR, and the high expression level in MDD and past month PTSD indicate an elevation in the level of systemic ER stress. Increased expression of $B i P$ suggests induction of ER stress response and activation of the UPR pathways (Bertolotti et al., 2000, Gulow et al., 2002). EDEM1 directly regulates ERassociated degradation (ERAD) by targeting misfolded glycoproteins for degradation in an $\mathrm{N}$-glycan-independent manner (Ron et al., 2011). Elevated expression of EDEM1 indicates induction of ER stress response and activation of the UPR that facilitates degradation of misfolded proteins in the ER (Molinari et al., 2003). In both MDD and PTSD, we found EDEM1 expression is elevated.

In contrast, in MDD--but not PTSD--CHOP and XBP1 expression are elevated. $C H O P$ expression is one downstream indicator of the $P E R K$-mediated UPR pathway (Zhang and Kaufman, 2004). Additionally, $C H O P$ is also recognized as a pro-apoptotic factor in ER stress-induced apoptosis (Zhang and Kaufman, 2004). Up-regulation of CHOP in MDD but not PTSD cases suggests that the PERK-mediated UPR pathway and ER stress-induced apoptosis in MDD may be more prevalent than that in PTSD. Finally, $X B P 1$ is a transcription factor that positively regulates $B i P, E D E M 1, C H O P$, and itself when the UPR is activated (Figure 2: adapted from Takayanagi et al., 2013). Notably, activation of $X B P 1$ is mediated through IREI $a$, the proximal UPR transducer (Zhang and Kaufman, 2004). Under ER stress conditions, IREI $a$ is activated to function as an RNase in order to alternately splice $X B P 1$ mRNA. Spliced XBP1 mRNA encodes a functional transcription factor that activates expression of the UPR target genes. Changes in the expression level of spliced $X B P 1$ can therefore reflect ER stress response and activation of the IREI $\alpha$-mediated UPR pathway, which regulate ERAD and expression of BiP. The elevated XBPI expression level in MDD may indicate a change in ER homeostasis and activation of the IRE $a$-mediated UPR pathway. 
ER stress may provide a mechanistic link between physical and mental diseases. Activation of ER stress and UPR related gene expression in MDD and PTSD may contribute to increased risk of a variety of physical diseases. Elevated ER stress genes in vascular tissue correlate with accelerated atherosclerotic plaque development in APOE deficient mice (Khan et al., 2009). Atherosclerosis results from a passive buildup of cholesterol in the artery wall (Libby et al., 2013). Diabetes increases atherosclerosis-related inflammation, and diabetic patients are twice as likely to have a heart attack or stroke. Khan and colleagues show that hyperglycemia-induced vascular ER stress response plays a role in diabetic atherosclerosis, and that inhibition of ER stress response attenuates diabetic atherosclerosis (Khan et al., 2009). There are significant comorbidities between diabetes and stress-related mental disorders such as MDD and PTSD (Anderson et al., 2001; Simon et al., 2006). It is plausible that increased expression levels of ER stress related genes in the peripheral blood in MDD may accelerate or facilitate atherosclerotic plaque development. Moreover, elevated ER stress may play a role in the risk of developing comorbid psychological and cardiovascular disease.

We recently showed that DNHS participants with lifetime depression showed evidence of increased inflammation (Uddin et al., 2010). In addition, we (Uddin et al., 2011) and others (Smith et al., 2011) have reported that participants with lifetime PTSD show epigenetic dysregulation in multiple immune-related genes, and an exaggerated immune response to a common pathogen (Uddin et al., 2010). General inflammation, oxidative stress, and calcium homeostasis share bi-directional interactions with the UPR pathway (Zhang, 2010). There are multiple lines of evidence suggesting that in addition to its roles in the ER stress response, the protein encoded by $X B P 1$ plays key roles in moderating innate immune response to pathogens. In the absence of $X B P I$ expression, pathogen induced inflammation results in an exaggerated ER stress response (Richardson et al., 2010). In the absence of $X B P 1$ expression, researchers report increased inflammation in the intestinal epithelium (Kaser et al., 2008). Conversely, XBP1 expression protects the host against ER stress caused by its own secretory immune response to infection (Richardson et al., 2010). Elevated rates of inflammation are a common phenotype associated with mental disorders including MDD and PTSD (Dantzer et al., 2008; Plantinga et al., 2013). It is possible that high expression levels of XBP1 in MDD cases may represent an adaptive response to elevated levels of inflammation associated with MDD. Unfortunately, up-regulation of $X B P 1$ also modulates lipid and glucose metabolism, which is associated with cardiovascular and metabolic diseases (Glimcher and Lee, 2009; Lee et al., 2012). Consequently, elevated ER stress conditions and activated $X B P 1$ expression may also play a role in the risk of developing comorbid psychological, cardiovascular and metabolic disease.

A non-invasive, quantitative clinical test of a single biomarker to aid in the diagnosis and treatment of MDD remains elusive (Lakhan et al, 2010). A variety of peripheral/serum growth factors, cytokines, hormones, and metabolic factors have been shown to be altered in MDD but are not specific to MDD, e.g. BDNF, IL-6, and TNF-a; a multivariate biomarker panel may in combination improve sensitivity and specificity for diagnosis and treatment of MDD (Schmidt, et al., 2011). Our data preliminarily suggest that ER stress pathway 
activation may represent an additional set of biomarkers that may contribute to diagnosis of MDD.

\section{STUDY LIMITATIONS}

Our study includes a minimum of four limitations that should be considered. First, the crosssectional analyses reported here leave us unable to determine whether the differences in expression were a consequence of MDD or PTSD or whether the differences in expression are indicative of biologic vulnerabilities that existed among the MDD-affected or PTSDaffected individuals before the onset of their disorder. Ongoing work using samples from this same longitudinal cohort may be informative regarding this distinction. Second, our focus on peripheral tissues limits the inferences that can be drawn about ER stress in the brain. Third, our sample size $(n=86)$ is modest. Nonetheless, we did observe statistically significant changes in expression (P $₫ 5.05$ ) across several members of the ER stress pathway, suggesting that the findings reported here are robust. Moreover, we applied an extremely stringent method to adjust for repeated measures which increases the probability of falsely rejecting a hypothesis, yet the expression data for MDD remained significantly different between cases and controls in each of the ER stress response genes that we tested. Finally, we assessed presence/absence of CVD-related conditions through self-report of a doctor's diagnosis. This may underreport CVD in our control sample. Given these results, replicating this work in independent cohorts is warranted.

\section{Conclusion}

Two stress-related mental disorders--MDD and PTSD--correlate with elevated expression levels of genes involved in the ER stress pathway. To our knowledge this is the first report associating PTSD or MDD with high systemic levels of ER stress. Patients diagnosed with either MDD or PTSD have an elevated risk of a variety of physical diseases. The ER stress response may contribute to this association, particularly with respect to heart disease and diabetes.

\section{Acknowledgments}

We would like to thank the participants of the DNHS for their time and generosity, this research would not be possible without their support. We would like to thank Rebecca M. Coulborn, Janie Slayden, and Helen Meier for overseeing DNHS specimen collection and coordinating the overall DNHS project. We also thank Amy Weckle, Alexis Pavle, Andrew Schultz, and Henriette Mair-Meijers for handling the DNHS specimen processing and laboratory technical assistance.

Role of the Funding Source

This study was supported by the National Institutes of Health (M.U., grant numbers DA022720, DA022720-S1, and RC1MH088283); the Wayne State University School of Medicine Postdoctoral Research Fellowship (L.N.); the American Association of Anatomists Post-doctoral Fellowship (L.N.); and the National Science Foundation (D.W., grant number BCS 0827546).

\section{References}

Anderson RJ, Freedland KE, Clouse RE, Lustman PJ. The prevalence of comorbid depression in adults with diabetes: a meta-analysis. Diabetes Care. 2001; 24:1069-78. [PubMed: 11375373] 
Back SH, Schroder M, Lee K, Zhang K, Kaufman RJ. ER stress signaling by regulated splicing: IRE1/ HAC1/XBP1. Methods. 2005; 35:395-416. [PubMed: 15804613]

Bertolotti A, Zhang Y, Hendershot LM, Harding HP, Ron D. Dynamic interaction of BiP and ER stress transducers in the unfolded-protein response. Nat Cell Biol. 2000; 2:326-32. [PubMed: 10854322]

Boscarino JA, Adams RE. Overview of findings from the World Trade Center Disaster Outcome Study: recommendations for future research after exposure to psychological trauma. Int J Emerg Ment Health. 2008; 10:275-90. [PubMed: 19278144]

Bown C, Wang JF, MacQueen G, Young LT. Increased temporal cortex ER stress proteins in depressed subjects who died by suicide. Neuropsychopharmacology. 2000; 22:327-32. [PubMed: 10693161]

Chae HJ, Kim HR, Xu C, Bailly-Maitre B, Krajewska M, Krajewski S, Banares S, Cui J, Digicaylioglu M, Ke N, Kitada S, Monosov E, Thomas M, Kress CL, Babendure JR, Tsien RY, Lipton SA, Reed JC. BI-1 regulates an apoptosis pathway linked to endoplasmic reticulum stress. Mol Cell. 2004; 15:355-66. [PubMed: 15304216]

Dantzer R, O’Connor JC, Freund GG, Johnson RW, Kelley KW. From inflammation to sickness and depression: when the immune system subjugates the brain. Nat Rev Neurosci. 2008; 9(1):46-56. [PubMed: 18073775]

Glembotski CC. Endoplasmic reticulum stress in the heart. Circ Res. 2007; 101:975-84. [PubMed: 17991891]

Glimcher LH, Lee AH. From sugar to fat: How the transcription factor XBP1 regulates hepatic lipogenesis. Ann N Y Acad Sci. 2009; 1173(S1):E2-9. [PubMed: 19751410]

Gold PW, Licinio J, Pavlatou MG. Pathological parainflammation and endoplasmic reticulum stress in depression: potential translational targets through the CNS insulin, klotho and PPAR-gamma systems. Mol Psychiatry. 2013; 18:154-65. [PubMed: 23183489]

Goldmann E, Aiello A, Uddin M, Delva J, Koenen K, Gant LM, Galea S. Pervasive exposure to violence and posttraumatic stress disorder in a predominantly African American Urban Community: the Detroit Neighborhood Health Study. J Trauma Stress. 2011; 24:747-51. [PubMed: 22144187]

Grippo AJ, Johnson AK. Biological mechanisms in the relationship between depression and heart disease. Neurosci Biobehav Rev. 2002; 26:941-62. [PubMed: 12667498]

Gulow K, Bienert D, Haas IG. BiP is feed-back regulated by control of protein translation efficiency. J Cell Sci. 2002; 115:2443-52. [PubMed: 12006628]

Hamada H, Suzuki M, Yuasa S, Mimura N, Shinozuka N, Takada Y, Nishino T, Nakaya H, Koseki H, Aoe T. Dilated cardiomyopathy caused by aberrant endoplasmic reticulum quality control in mutant KDEL receptor transgenic mice. Mol Cell Biol. 2004; 24:8007-17. [PubMed: 15340063]

Hunsberger JG, Machado-Vieira R, Austin DR, Zarate C, Chuang DM, Chen G, Reed JC, Manji HK. Bax inhibitor 1, a modulator of calcium homeostasis, confers affective resilience. Brain Res. 2011; 1403:19-27. [PubMed: 21718971]

Jiao Q, Takeshima H, Ishikawa Y, Minamisawa S. Sarcalumenin plays a critical role in age-related cardiac dysfunction due to decreases in SERCA2a expression and activity. Cell Calcium. 2012; 51:31-9. [PubMed: 22119571]

Kaser A, Lee AH, Franke A, Glickman JN, Zeissig S, Tilg H, Nieuwenhuis EE, Higgins DE, Schreiber S, Glimcher LH, Blumberg RS. XBP1 links ER stress to intestinal inflammation and confers genetic risk for human inflammatory bowel disease. Cell. 2008; 134:743-56. [PubMed: 18775308]

Kaster MP, Gadotti VM, Calixto JB, Santos AR, Rodrigues AL. Depressive-like behavior induced by tumor necrosis factor-alpha in mice. Neuropharmacology. 2012; 62:419-26. [PubMed: 21867719]

Keyes KM, McLaughlin KA, Demmer RT, Cerdá M, Koenen KC, Uddin M, Galea S. Potentially traumatic events and the risk of six physical health conditions in a population-based sample. Depress Anxiety. 2013; 30(5):451-60. [PubMed: 23495094]

Khan MI, Pichna BA, Shi Y, Bowes AJ, Werstuck GH. Evidence supporting a role for endoplasmic reticulum stress in the development of atherosclerosis in a hyperglycaemic mouse model. Antioxid Redox Signal. 2009; 11:2289-98. [PubMed: 19548776] 
Kroenke K, Spitzer RL, Williams JB. The PHQ-9: validity of a brief depression severity measure. J Gen Intern Med. 2001; 16:606-13. [PubMed: 11556941]

Kubzansky LD, Koenen KC, Spiro A 3rd, Vokonas PS, Sparrow D. Prospective study of posttraumatic stress disorder symptoms and coronary heart disease in the Normative Aging Study. Arch Gen Psychiatry. 2007; 64:109-16. [PubMed: 17199060]

Lee JS, Mendez R, Heng HH, Yang ZQ, Zhang K. Pharmacological ER stress promotes hepatic lipogenesis and lipid droplet formation. Am J Transl Res. 2012; 4(1):102-13. [PubMed: 22347525]

Libby P, Lichtman AH, Hansson GK. Immune effector mechanisms implicated in atherosclerosis: from mice to humans. Immunity. 2013; 38(6):1092-104. [PubMed: 23809160]

Liu Y, Yang N, Hao W, Zhao Q, Ying T, Liu S, Li Q, Liang Y, Wang T, Dong Y, Ji C, Zuo P. Dynamic proteomic analysis of protein expression profiles in whole brain of Balb/c mice subjected to unpredictable chronic mild stress: implications for depressive disorders and future therapies. Neurochem Int. 2011; 58:904-13. [PubMed: 21349309]

Livak KJ, Schmittgen TD. Analysis of relative gene expression data using real-time quantitative PCR and the 2(-Delta Delta C(T)) Method. Methods. 2001; 25:402-8. [PubMed: 11846609]

Meares GP, Mines MA, Beurel E, Eom TY, Song L, Zmijewska AA, Jope RS. Glycogen synthase kinase-3 regulates endoplasmic reticulum (ER) stress-induced CHOP expression in neuronal cells. Exp Cell Res. 2011; 317:1621-8. [PubMed: 21356208]

Minamino T, Kitakaze M. ER stress in cardiovascular disease. J Mol Cell Cardiol. 2010; 48:1105-10. [PubMed: 19913545]

Mines MA, Yuskaitis CJ, King MK, Beurel E, Jope RS. GSK3 influences social preference and anxiety-related behaviors during social interaction in a mouse model of fragile $\mathrm{X}$ syndrome and autism. PLoS One. 2010; 5:e9706. [PubMed: 20300527]

Molinari M, Calanca V, Galli C, Lucca P, Pagnetti P. Role of EDEM in the release of misfolded glycoproteins from the calnexin cycle. Science. 2003; 299:1397-1400. [PubMed: 12610306]

Myoishi M, Hao H, Minamino T, Watanabe K, Nishihira K, Hatakeyama K, Asada Y, Okada K, Ishibashi-Ueda H, Gabbiani G, Bochaton-Piallat ML, Mochizuki N, Kitakaze M. Increased endoplasmic reticulum stress in atherosclerotic plaques associated with acute coronary syndrome. Circulation. 2007; 116:1226-33. [PubMed: 17709641]

O'Connor JC, Andre C, Wang Y, Lawson MA, Szegedi SS, Lestage J, Castanon N, Kelley KW, Dantzer R. Interferon-gamma and tumor necrosis factor-alpha mediate the upregulation of indoleamine 2,3-dioxygenase and the induction of depressive-like behavior in mice in response to bacillus Calmette-Guerin. J Neurosci. 2009; 29:4200-9. [PubMed: 19339614]

Plantinga L, Bremner JD, Miller AH, Jones DP, Veledar E, Goldberg J, Vaccarino V. Association between posttraumatic stress disorder and inflammation: a twin study. Brain Behav Immun. 2013; 30:125-32. [PubMed: 23379997]

Reddy RK, Mao C, Baumeister P, Austin RC, Kaufman RJ, Lee AS. Endoplasmic reticulum chaperone protein GRP78 protects cells from apoptosis induced by topoisomerase inhibitors: role of ATP binding site in suppression of caspase-7 activation. J Biol Chem. 2003; 278(23):20915-24. [PubMed: 12665508]

Richardson CE, Kooistra T, Kim DH. An essential role for XBP-1 in host protection against immune activation in C. elegans. Nature. 2010; 463:1092-5. [PubMed: 20182512]

Ron E, Shenkman M, Groisman B, Izenshtein Y, Leitman J, Lederkremer GZ. Bypass of glycandependent glycoprotein delivery to ERAD by up-regulated EDEM1. Mol Biol Cell. 2011; 22(21): 3945-3954. [PubMed: 21917589]

Schmittgen TD, Livak KJ. Analyzing real-time PCR data by the comparative C(T) method. Nat Protoc. 2008; 3:1101-8. [PubMed: 18546601]

Simon GE, Von Korff M, Saunders K, Miglioretti DL, Crane PK, van Belle G, Kessler RC. Association between obesity and psychiatric disorders in the US adult population. Arch Gen Psychiatry. 2006; 63:824-30. [PubMed: 16818872]

Smith A, Conneely K, Kilaru V, Mercer K, Weiss T, Bradley B, Tang Y, Gillespie C, Cubells J, Ressler K. Differential immune system DNA methylation and cytokine regulation in post- 
traumatic stress disorder. Am J Med Genet B Neuropsychiatr Genet. 2011; 156B:700-8. [PubMed: 21714072]

So J, Warsh JJ, Li PP. Impaired endoplasmic reticulum stress response in B-lymphoblasts from patients with bipolar-I disorder. Biol Psychiatry. 2007; 62:141-7. [PubMed: 17217928]

Sun Y, Liu G, Song T, Liu F, Kang W, Zhang Y, Ge Z. Upregulation of GRP78 and caspase-12 in diastolic failing heart. Acta Biochim Pol. 2008; 55:511-6. [PubMed: 18787714]

Takayanagi S, Fukuda R, Takeuchi Y, Tsukada S, Yoshida K. Gene regulatory network of unfolded protein response genes in endoplasmic reticulum stress. Cell Stress Chaperones. 2013; 18(1):1123. [PubMed: 22802018]

Thuerauf DJ, Marcinko M, Gude N, Rubio M, Sussman MA, Glembotski CC. Activation of the unfolded protein response in infarcted mouse heart and hypoxic cultured cardiac myocytes. Circ Res. 2006; 99:275-82. [PubMed: 16794188]

Uddin M, Aiello AE, Wildman DE, Koenen KC, Pawelec G, de Los Santos R, Goldmann E, Galea S. Epigenetic and immune function profiles associated with posttraumatic stress disorder. Proc Natl Acad Sci U S A. 2010; 107:9470-5. [PubMed: 20439746]

Uddin M, Koenen KC, Aiello AE, Wildman DE, de los Santos R, Galea S. Epigenetic and inflammatory marker profiles associated with depression in a community-based epidemiologic sample. Psychol Med. 2011; 41:997-1007. [PubMed: 20836906]

Wang JF, Bown C, Young LT. Differential display PCR reveals novel targets for the mood-stabilizing drug valproate including the molecular chaperone GRP78. Mol Pharmacol. 1999; 55(3):521-7. [PubMed: 10051536]

Zhang K. Integration of ER stress, oxidative stress and the inflammatory response in health and disease. Int J Clin Exp Med. 2010; 3:33-40. [PubMed: 20369038]

Zhang K, Kaufman RJ. Signaling the unfolded protein response from the endoplasmic reticulum. J Biol Chem. 2004; 279:25935-8. [PubMed: 15070890]

Zhang K, Kaufman RJ. From endoplasmic-reticulum stress to the inflammatory response. Nature. 2008; 454:455-62. [PubMed: 18650916] 

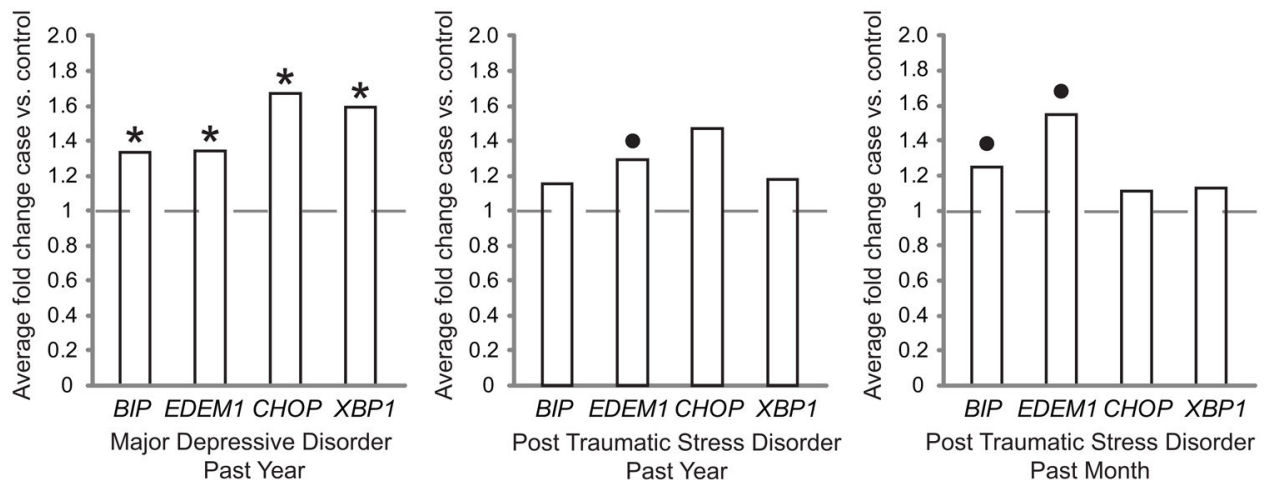

Figure 1.

Average fold change between cases and controls for Major Depressive Disorder and Post Traumatic Stress Disorder.

The closed circle indicates a statistically significant increase in expression in cases compared with controls at (uncorrected $\mathrm{p}<0.05$ ). The star symbol indicates a statistically significant increase in expression in cases compared with controls at $(\mathrm{P}<0.0125$; i.e. a $₫ 0.05$ adjusted for multiple comparisons with the Bonferroni correction). ER stress related gene expression is elevated in MDD and PTSD. 

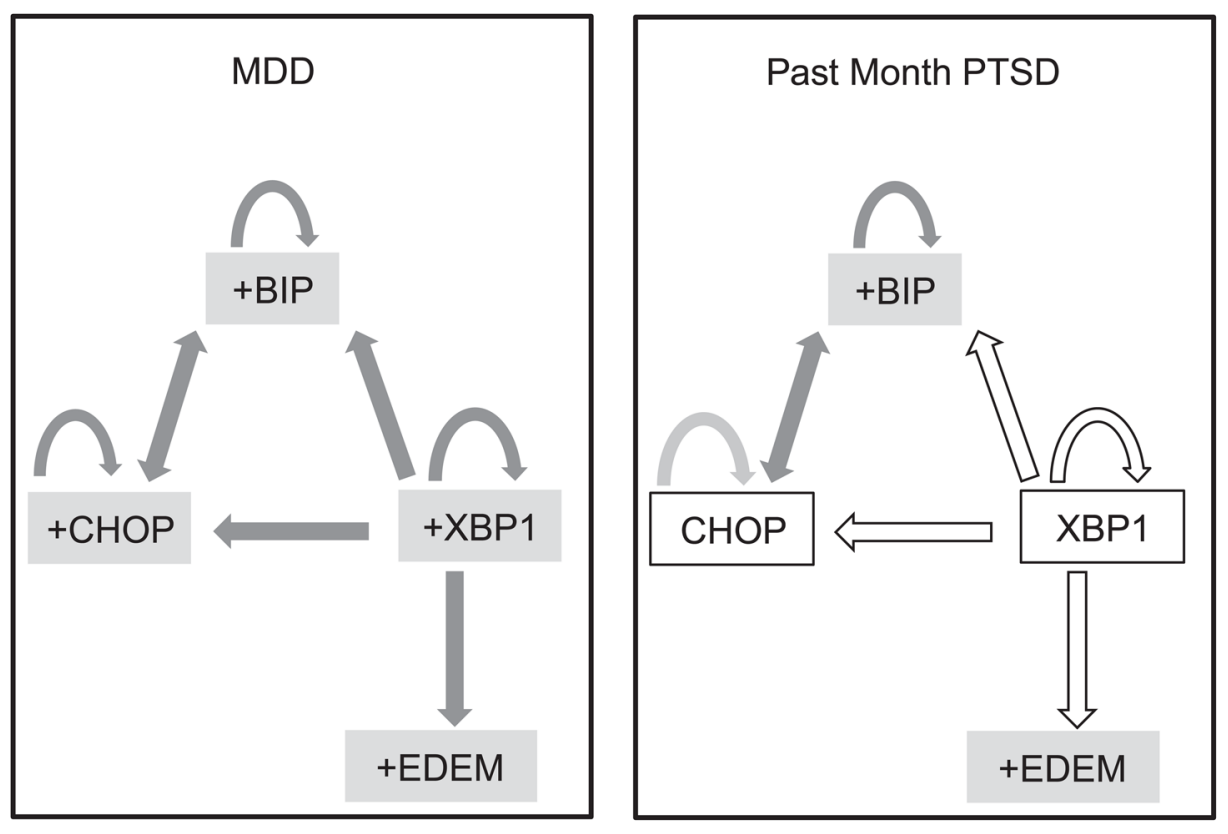

Figure 2.

ER stress transcriptional network and dysregulation in Major Depressive Disorder and Past Month Post Traumatic Stress Disorder.

Each grey arrow indicates a transcriptional target of a significantly up regulated gene. Each white arrow indicates aspects of the ER stress transcriptional network apparently unaffected in MDD or past month PTSD. Each of the genes tested are a transcriptional target of $X B P 1$, therefore the elevated expression observed in MDD may represent a change in ER stress homeostasis. 


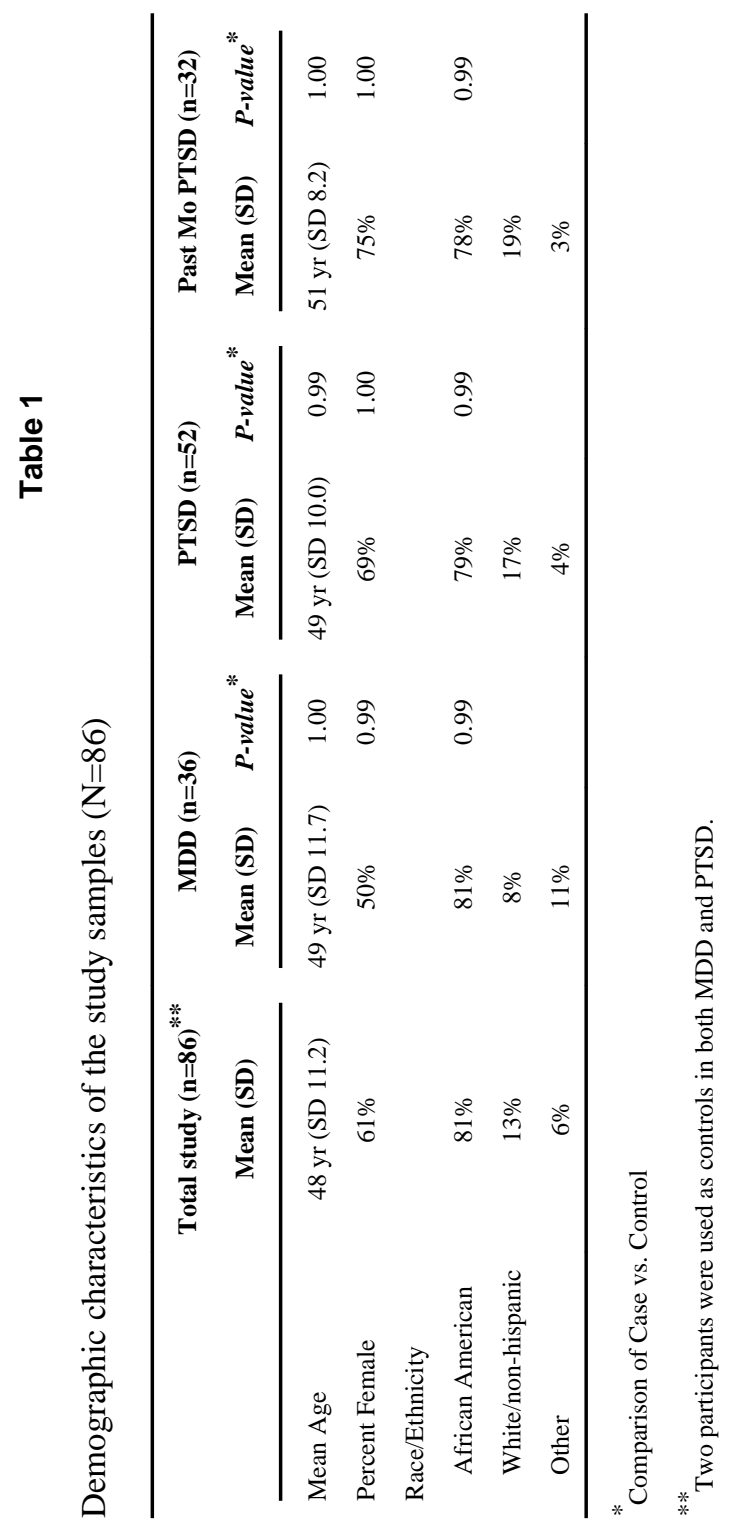




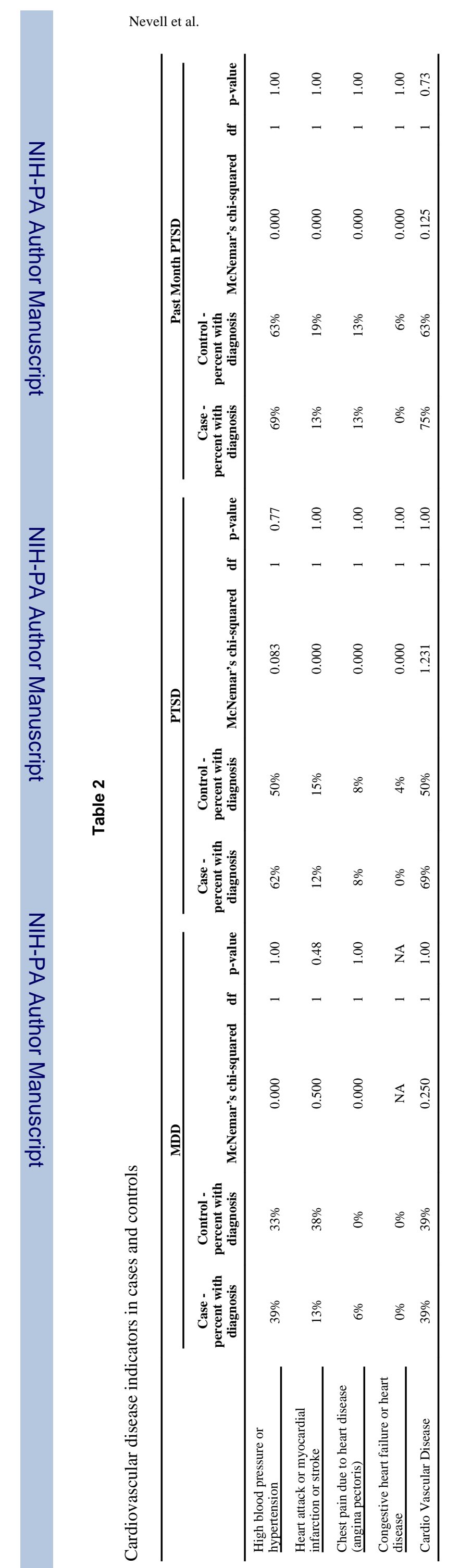

Page 17 